Z. Phys. B - Condensed Matter 64, 19-23 (1986)

\title{
Non-Ohmic Conduction in Doped Polyacetylene at Low Temperatures
}

\author{
H. Chaabane, E. Ettlinger, and W. Schoepe \\ Institut für Angewandte Physik, Universität Regensburg, \\ Federal Republic of Germany
}

Received March 27, 1986

Dedicated to K. Dransfeld on the occasion of his 60th birthday

\begin{abstract}
The electrical conductivity of a iodine-doped polyacetylene is measured as a function of the electric field $\mathscr{E}$ at temperatures between $4 \mathrm{~K}$ and $0.3 \mathrm{~K}$. We find that after an initial non-linear behavior $\sigma$ increases linearly with $\mathscr{E}$ in agreement with a theoretical description based on variable-range hopping conduction. The non-linear rise at low fields depends on the iodine concentration. In heavily doped samples the increase is small and varies as $\mathscr{E}^{2}$, whereas in less conductive samples a large change is observed at $0.3 \mathrm{~K}$ which varies approximately as $\log \mathscr{E}$ for fields from $1 \mathrm{~V} / \mathrm{m}$ to $150 \mathrm{~V} / \mathrm{m}$.
\end{abstract}

\section{Introduction}

The high electrical conductivity of doped polyacetylene is of considerable interest both from a fundamental point as well as for possible applications. A large number of experimental and theoretical investigations have been made to reveal the electronic transport mechanism [1]. Among these are measurements of the $d c$ conductivity as a function of temperature $T$, magnetic field $B$, and electric field $\mathscr{E}$ on samples of different fabrication, of various dopant ions, and different dopant concentrations. In spite of all this work, our present understanding of the charge transport is still far from being complete.

The temperature dependence of the conductivity $\sigma$ of lightly doped samples can be described by variable-range hopping (VRH) known from disordered semiconductors [2]: $\sigma \propto \exp -\left(T_{0} / T\right)^{1 / n}$, where $n$ depends on the density of states (DOS) at the Fermi level. In general, one has $n=4$ (constant DOS) though fits to the data may also be made with $n=2$ (parabolic DOS). The quantity $T_{0}$ depends on the localization length and on the DOS. What is actually hopping, however, remains open: holes, polarons and solitons have been considered. Alternatively, tunneling conduction between microscopic metallic islands has been suggested [3] and can also be fit successfully to the data, in particular at higher dopant concentrations where VRH fails to describe the temperature dependence of $\sigma$. Finally, at very high doping levels $\sigma$ approaches metallic values and decreases only very little, even down to ultra-low temperatures [4]. No satisfactory model for this behavior is available. More detailed information may be drawn from the magnetic field dependence of $\sigma$. For low dopant concentration a large positive magnetoresistance (MR) is observed below $4 \mathrm{~K}$ which varies as $B^{2}$ at low fields and tends to saturate in high fields below $1 \mathrm{~K}$ [5]. This result is attributed to orbital shrinking of the localized wavefunction in the magnetic field. From a fit to the data the localization length and the DOS can be determined. In most samples the DOS is found to be parabolic: $N(E)=N_{0} \Delta E^{2}$, where $\Delta E=E-E_{F}$. In order to obtain a saturation a second transport mechanism must be postulated which shortens out the vanishing VRH conduction in large fields. Other models based on spin effects are also being discussed [6], though no detailed fit to the data has been made yet. With increasing doping level the MR continuously decreases and changes sign. At $4 \mathrm{~K}$ a small negative $\mathrm{MR}$ is observed [7] which becomes positive below $1 \mathrm{~K}$ and has a maximum near 2.5 Tesla. Finally, in very metallic samples only a negative $M R$ is ob- 
served even down to very low temperatures $[4,8]$. The negative MR may be due to a spin-dependent localization length $[7,9]$ enhancing charge transport for the spin orientation whose localization length is increased by the field. Again, also other models may be envisaged.

The electric field dependence of $\sigma$ is completely unclear. Different non-Ohmic behavior has been observed [10-12]. While $\sigma$ is always found to increase with the field $\mathscr{E}$ a model which can describe the experiments in a satisfactory way does not exist, in particular when in addition the $T$ and $B$ dependence of $\sigma$ are included. Ultimately, it would be most desirable to have a complete theoretical description of $\sigma(T, B, \mathscr{E})$. We therefore have made an effort to investigate non-Ohmic effects in iodine-doped polyacetylene at low temperatures. To a large extent our data can be discussed in terms of VRH, especially for fields $\mathscr{E}$ above $50 \mathrm{~V} / \mathrm{m}$. However, most interestingly we also find a large increase of $\sigma$ at low temperatures which varies logarithmically with $\mathscr{E}$ down to less than $1 \mathrm{~V} / \mathrm{m}$ and for which we have no immediate explanation.

\section{Experimental}

In order to obtain reliable results for the conductivity several experimental requirements have to be met. Firstly, because of the temperature dependence of $\sigma$ the measuring current must be pulsed. The pulses have to be short enough to avoid heating during the pulse. When this becomes too difficult, only the initial change of $\sigma$ at the beginning of the pulse (few microseconds) can be attributed to the non-Ohmic conduction. The duty cycle must then be low enough to prevent an average temperature increase. Immersing the sample into the helium-3 bath is necessary for thermal stability. Secondly, a fourprobe method removes undesired effects from contact resistances between the electrodes and the sample. Furthermore, to measure a well defined $\sigma(\mathscr{E})$ a homogeneous field $\mathscr{E}$ is required across the sample between the voltage electrodes. Below we describe our set-up.

For the resistance measurements we use a pulsed four-terminal bridge with a precision instrumentation amplifier in its diagonal. The amplified signal is fed to a transient recorder which is controlled by a microcomputer. Typical pulse widths are $50 \mu$ s and repetition rates are $0.2-1 \mathrm{~Hz}$. On account of this low duty cycle we avoid an average heating effect. Heating during the pulse is observed at high current levels and will be discussed in the Appendix. To establish a homogeneous electric field across the vol- tage electrodes the current contacts cover the whole width of the sample so that the current is uniformly distributed. Spacing between the current probes is a factor of 10 larger than that between the voltage probes. The samples were polymerized by the Stuttgart group and doped by us with iodine.

\section{Results and Discussion}

Typical results of differently doped samples are shown in Figs. 1 and 2 where the relative change of the conductivity $\Delta \sigma / \sigma_{0}$ is displayed as a function of the electric field $\mathscr{E}$ at various constant temperatures. Both samples show two regimes of electrical field dependence: a non-linear initial increase of the conductivity at low fields, which then changes into a linear behavior in moderate fields.

Considering the linear part of $\Delta \sigma / \sigma_{0}$ we find fair agreement with predictions of the VRH model [13] and related work on semiconductors $[14,15]$. The electric field reduces the energy barrier between two

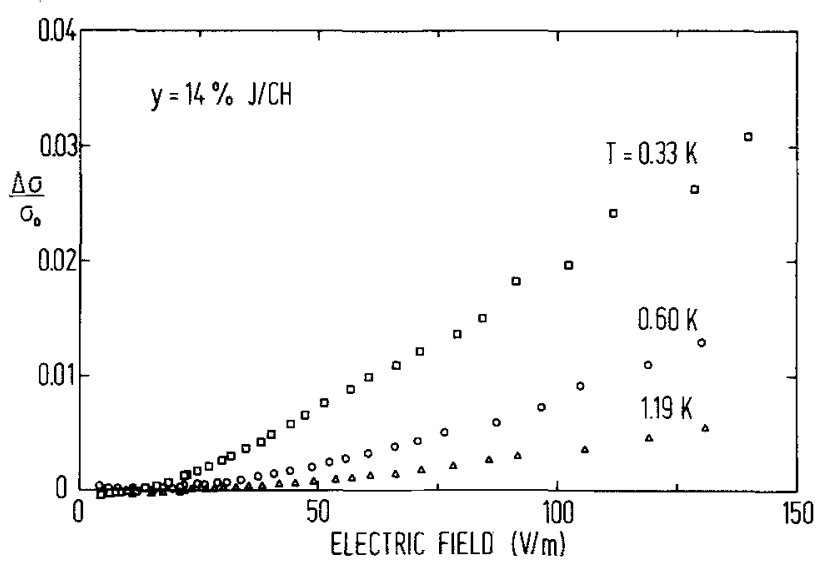

Fig. 1. Relative change of the electrical conductivity versus the electrical field of sample $\left(\mathrm{CHI}_{0.14}\right)_{x}$

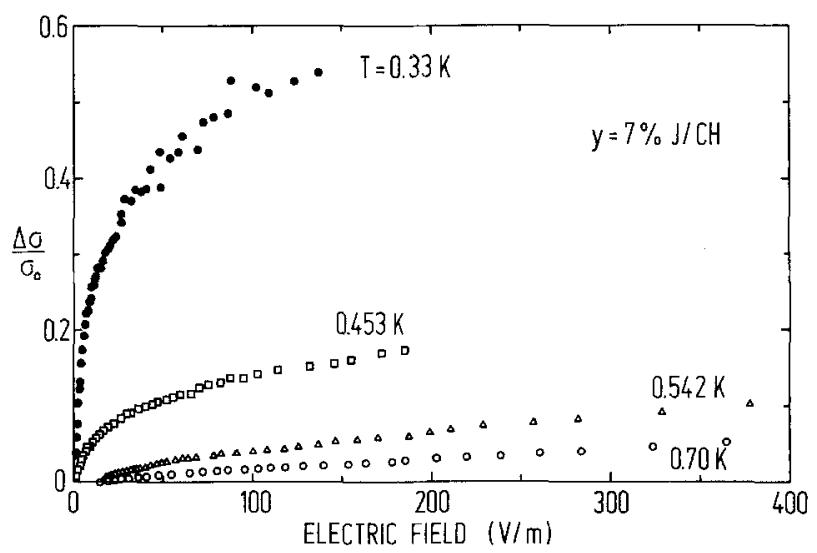

Fig. 2. Relative change of the electrical conductivity versus the electrical field of sample $\left(\mathrm{CHI}_{0.07}\right)_{x}$ 


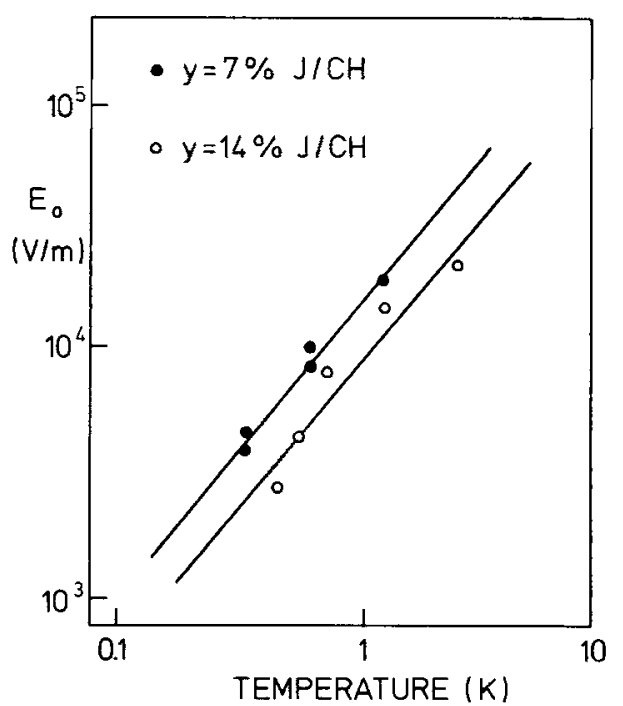

Fig. 3. Characteristic fields of both samples as a function of the temperature. The solid lines are least squares fits to a power law $T^{x}$ with $x=1.2$

Table 1. Temperature dependence of the characteristic field $\mathscr{E}_{0}(T)$

\begin{tabular}{lllll}
\hline Theory & & & Experiment \\
\hline$N=$ const & $N=N_{0} \cdot\left(E-E_{F}\right)^{2}$ & & $7 \% I^{-}$ & $14 \% I^{-}$ \\
\hline$T^{1.25}$ & $T^{1.5}$ & $T^{1.2}$ & $T^{1.2}$
\end{tabular}

sites by an amount $e \mathscr{E} l$, where $l$ is the hopping length. One obtains therefore the following formula:

$\sigma(\mathscr{E})=\sigma(0) \exp \left(\mathscr{E} / \mathscr{E}_{0}(T)\right)$

where

$\mathscr{E}_{0}(T)=k T / e l$.

The hopping length $l$ depends on the DOS $N(E)$. One finds $l \propto T^{-1 / 4}$ and hence $\mathscr{E}_{0} \propto T^{5 / 4}$ for a constant DOS and for a parabolic DOS $l \propto T^{-1 / 2}$ and $\mathscr{E}_{0} \propto T^{3 / 2}$. For small values of $\mathscr{E} / \mathscr{E}_{0}$ we can expand (1):

$\sigma(\mathscr{E})=\sigma(0) \cdot\left(1+\mathscr{E} / \mathscr{E}_{0}\right)$

and we finally obtain the expression

$\Delta \sigma / \sigma_{0}=\mathscr{E}_{\mathscr{E}} / \mathscr{E}_{0}(T)$

which describes the observed linear behavior. From the inverse of the slope of our graphs we get the absolute values of the characteristic fields $\mathscr{E}_{0}(T)$ and their temperature dependences. The results for two of our samples $\left(7 \% I^{-}\right.$and $\left.14 \% I^{-}\right)$are shown in Fig. 3 and compiled in Table 1 together with the
Table 2. Temperature dependence of the hopping length of a jump

\begin{tabular}{lrl}
\hline & Sample & \\
\cline { 2 - 3 } & $7 \% I^{-}$ & $14 \%^{-}$ \\
\hline hopping length & & \\
at $1 \mathrm{~K}$ & $59 \AA$ & $45 \AA$ \\
at $0.6 \mathrm{~K}$ & $114 \AA$ & $63 \AA$ \\
\hline
\end{tabular}

above theoretical predictions for purely constant and parabolic DOS.

From the absolute value of the characteristic fields $\mathscr{E}_{0}(T)$ one can calculate the hopping length from (2) (see Table 2). In comparison with our former magnetoresistance data [7] this hopping length is smaller by a factor of 7 . Several authors suggest that the field changes the percolation paths in such a way that the above quantity $l$ in (1) is only a fraction $C<1$ of the actual hopping length. Theoretical estimations of the absolute value of $C$ vary from $C$ $=0.17$ (Pollak and Riess [16]), $0.19<C<0.46$ (Levin and Shklovskii [13] up to $C=0.8$ (Hill [17]). Within these bounds our values for $l$ (see Table 2) are consistent with our former analysis of the MR data.

The initial non-linear increase of $\sigma$ at low fields depends on both the temperature and the doping level. It is found to vary as $\mathscr{E}^{2}$ at all temperatures for the more conductive sample (see Fig. 1) whereas at low dopant concentration it is observed only at high temperature $(4.2 \mathrm{~K})$. This behavior might therefore be characteristic for short hopping lengths. A quadratic variation of $\sigma$ has also been observed in semiconductors at very low fields [14] in the transition regime between purely Ohmic and exponential conduction. It is not surprising that this transition is smooth. There are also theoretical arguments for an initial $\mathscr{E}^{2}$-deviation from Ohm's law $[17,18]$. Because our observed changes of $\sigma$ are less than $1 \%$ in this regime we do not want to analyze them in more detail here.

A much larger non-linear increase of $\sigma$ occurs in the less conductive sample at low temperatures (see Fig. 2). At $0.33 \mathrm{~K} \sigma$ changes by more than $50 \%$ when the field is increased from about $1 \mathrm{~V} / \mathrm{m}$ to $150 \mathrm{~V} / \mathrm{m}$. The increase follows a $\log \left(\mathscr{E} / \mathscr{E}_{0}\right)$ behavior with a threshold field $\mathscr{E}_{0}$ of only $0.8 \mathrm{~V} / \mathrm{m}$ (see Fig. 4) and depends strongly on the temperature. For a fixed field $\mathscr{E}=100 \mathrm{~V} / \mathrm{m}$ (i.e. at the end of the nonlinear regime) $\Delta \sigma / \sigma_{0}$ varies approximately as $T^{-4.5}$ between $0.33 \mathrm{~K}$ and $0.70 \mathrm{~K}$. So far, we have no explanation for this result. An extension of our work to even lower temperatures may be helpful for an identification of the origin of this behavior. It should be mentioned, however, that an unexplained loga- 


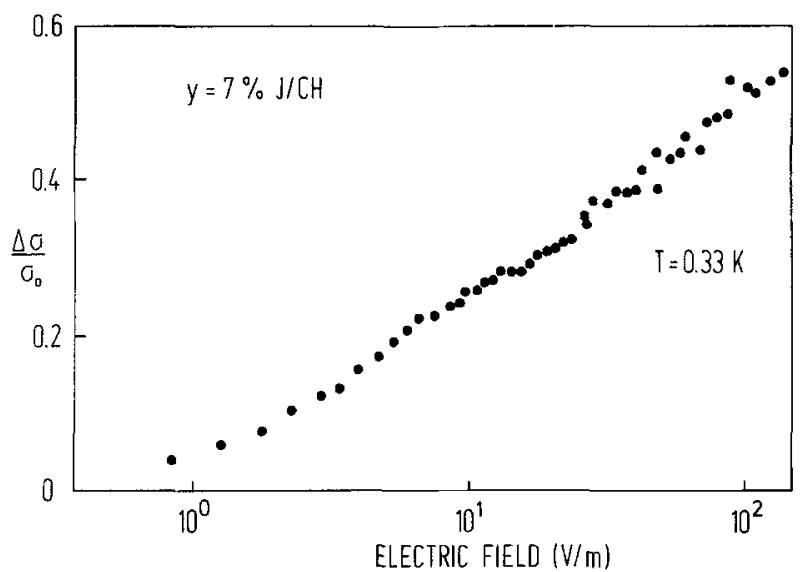

Fig. 4. Relative change of the conductivity of sample $\left(\mathrm{CHI}_{0.07}\right)_{x}$ versus the logarithm of the electrical field at a temperature of $0.33 \mathrm{~K}$

rithmic field dependence has been observed earlier at much higher temperatures [11] with threshold fields down to about $1 \mathrm{~V} / \mathrm{m}$ at $15 \mathrm{~K}$. The slopes of the logarithmic term, though, were independent of temperature, in contrast to our observation, and about a factor of 2 smaller than our value of $0.33 \mathrm{~K}$. Therefore, it is not clear whether we are dealing with the same effect.

In summary we find that the linear part of the non-Ohmic conductivity is consistent with VRH. Concerning the logarithmic regime, however, we feel that further work is required for a better understanding of the $\mathrm{dc}$ conductivity of doped polyacetylene. The situation will presumably improve considerably when experimental results from highly oriented samples become available, which are not influenced by the complicated fibrillar morphology as might be the case here.

It is a pleasure to thank $\mathrm{S}$. Roth for supplying the samples and for helpful discussions. H.C. acknowledges support from the Friedrich-Ebert Foundation. E.E. is a recipient of a Bavarian Graduate Scholarship.

\section{Appendix: Heating Effects}

At large current amplitudes and long pulse widths heating of the sample can easily be observed. The flat plateau of $\Delta R$ changes to a linear time dependence indicating an additional drop of the resistance because of heating, see Fig. 5 .

For even longer pulses the drop $\Delta R_{1}$ ceases to grow linearly and eventually levels off. In the following we analyze the linear growth $\Delta R_{1}=\dot{R} \Delta t$. The measurements were performed with the sample in

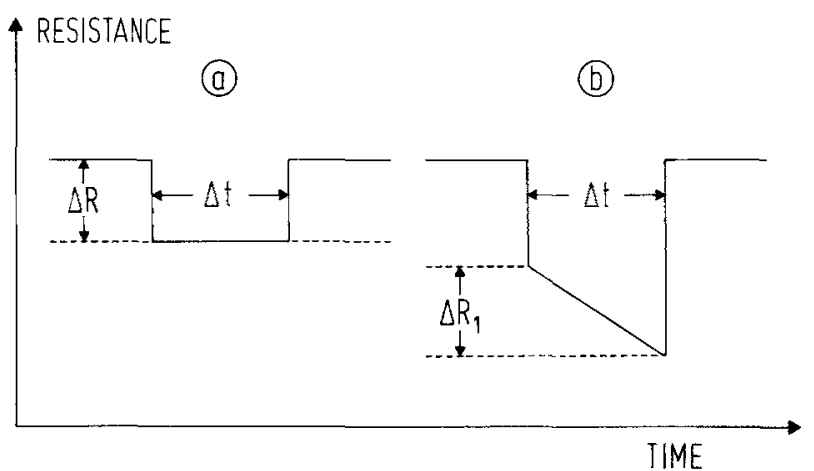

Fig. 5a and b. Time dependence of the resistance during the current pulse: a without heating effects; $b$ with heating effects a linear drop $\Delta R_{1}$ occurs due to the temperature dependence of the conductivity

vacuum (largest heating), in ${ }^{3} \mathrm{He}$ gas, and in liquid ${ }^{3} \mathrm{He}$ (smallest heating).

Heat is generated at a rate $I^{2} R$, where $I$ is the current and $R$ the sample resistance. The temperature therefore changes at a rate $\dot{T}=I^{2} R / C$, where $C$ is a specific heat. For small temperature changes $\Delta T$ we approximate the known $R(T)$ curve linearly: $\Delta R$ $=a \Delta T$, where $a\left(T_{0}\right)$ is the slope at the temperature $T_{0} \gg \Delta T$. We therefore have

$\dot{R}=a I^{2} R / C$.

By plotting the measured $\dot{R}$ against $I^{2}$ we obtain a straight line from which we determine $C$.

At $4.2 \mathrm{~K}$ we find $C=3.0 \cdot 10^{-6} \mathrm{~J} / \mathrm{K}$ when the sample is in vacuum. Estimating the mass of the sample between the voltage probes from the weight of the entire sample and the geometry $\left(2.3 \cdot 10^{-4} \mathrm{~g} / \mathrm{mm}^{2}\right)$ to be about $10^{-5}$ mol we get a molar specific heat of $0.3 \mathrm{~J} / \mathrm{mol} \mathrm{K}$ which is of the expected order of magnitude when compared with Ref. 19.

Introducing some ${ }^{3} \mathrm{He}$ gas into the measuring cell reduces $\dot{R}$ by $60 \%$ and hence increases C. We believe this to be due to the specific heat of the gas between the fibrils. We therefore lower the temperature to $0.45 \mathrm{~K}$ and imerse the sample completely into liquid ${ }^{3} \mathrm{He}$. Repeating the measurements and the above analysis we obtain a specific heat $C$ $=8.6 \cdot 10^{-6} \mathrm{~J} / \mathrm{K}$, larger than before. Assuming a filling factor of $50 \%$ we estimate to have $3 \cdot 10^{-6} \mathrm{~mol}$ ${ }^{3} \mathrm{He}$ between the fibrils. From the molar specific heat of liquid ${ }^{3} \mathrm{He} C \approx 3 \mathrm{~J} / \mathrm{mol} \cdot \mathrm{K}$ at $0.45 \mathrm{~K} \mathrm{[20]}$ we in fact can explain the above heating rate (the specific heat of the sample being negligible compared to that of the liquid). The interesting conclusion from this result is that polyacetylene has an exceedingly good thermal contact to the liquid probably because of its open fibrillar structure. 


\section{References}

1. Chien, J.C.W.: Polyacetylene: Chemistry, physics, and material science, pp. 479-565. Orlando: Academic Press 1984

2. Shklovskii, B.I., Efros, A.L.: Electromic properties of doped semiconductors. In: Springer Series in Solid-State Sciences. Vol. 45. Berlin, Heidelberg, New York: Springer 1984

3. Sheng, P.: Phys. Rev. B21, 2180 (1980)

4. Gould, C.M., Bates, D.M., Bozler, H.M., Heeger, A.J., Druy, M.A., MacDiarmid, A.G.: Phys. Rev. B23, 6820 (1981)

5. Ettlinger, E., Schoepe, W., Monkenbusch, M., Wieners, G.: Solid State Commun. 49, 107 (1984)

6. Kurobe, A., Kamimura, H.: J. Phys. Soc. Jpn. 51, 1904 (1982) Ovchinnikov, A.A., Pronin, K.A.: Dokl. Akad. Nauk SSSR 276, 594 (1984) [Sov. Phys. Dokl. 29, 5 (1984)]

7. Ettlinger, E., Ose, W., Schoepe, W.: Mol. Cryst. Liq. Cryst. 117, 173 (1985)

8. Kwak, F.J., Clarke, T.C., Greene, R.L., Street, G.B.: Solid State Commun. 31, 355 (1979)

9. Fukuyama, H., Yosida, K.: J. Phys. Soc. Jpn. 46, 102 (1979)

10. Mortensen, K., Thewalt, M.L.W., Tomkiewicz, Y., Clarke, T.C., Street, G.B.: Phys. Rev. Lett. 45, 490 (1980)

11. Epstein, A.J., Gibson, H.W., Chaikin, P.M., Clark, W.G., Grïner, G.: Phys. Rev. Lett. 45, 1730 (1980)
12. Philipp, A., Mayr, W., Seeger, K.: Solid State Commun. 43, 857 (1982)

13. Levin, E.I., Shklovskii, B.I.: Fiz. Tekh. Poluprovodn. 18, 856 (1984) [Sov. Phys. Semicond. 18, 534 (1984)]

14. Redfield, D.: Adv. Phys. 24, 463 (1975)

15. Zavaritskaya, E.I.: Pis'ma Zh. Eksp. Teor. Fiz. 41, 231 (1985) [Sov. Phys. JETP Lett. 41, 279 (1985)]

16. Pollak, M., Riess, I.: J. Phys. C9, 2339 (1976)

17. Hill, R.M.: Philos. Mag. 24, 1307 (1971)

18. Apsley, N., Hughes, H.P.: Philos. Mag. 31, 1327 (1975)

19. Schweizer, R.J., Menke, K., Göhring, W., Roth, S.: Mol. Cryst. Liq. Cryst. 117, 181 (1985)

20. Lounasmaa, O.V.: Experimental principles and methods below $1 \mathrm{~K}$, p. 280 . London and New York: Academic Press 1974

H. Chaabane

E. Ettlinger

W. Schoepe

Institut für Angewandte Physik

Universität Regensburg

Universitätsstrasse 31

D-8400 Regensburg

Federal Republic of Germany 